\title{
Gestion d'un réservoir pour la régularisation des débits
}

\author{
X. Yang $(*)$, E. Parent $(*)$, C. Michel $\left({ }^{* *}\right)$, P. A. Roche $\left({ }^{*}\right)$
}

\section{Introduction}

La gestion des réservoirs d'eau de surface pour la régularisation de la ressource est un problème étudié depuis près d'un siècle mais conserve une actualité certaine du fait de la complexité des objectifs poursuivis et de la difficulté à prévoir les écoulements futurs. Avec un seul réservoir et un objectif double d'écrêtement de crues et de soutien d'étiage, le problème demeure intéressant car on manque encore de méthodes simples et fiables qui ne préjugent pas de la structure mathématique de la règle de gestion.

La démarche développée dans ce document s'inspire de travaux antérieurs (MIQUEL J., Roche P. A., 1983, Goussebaille J., MiQuel J., Roche P. A., 1986) où l'on associait une méthode de programmation dynamique en avenir certain à une génération de nombreux scénarios pour prendre en compte l'aspect aléatoire des écoulements à venir. Pour obtenir une méthode d'emploi encore plus rapide, et compte tenu de la simplicité des objectifs visés on propose une méthode où l'optimisation repose sur la méthode du "fil-tendu" (VARLET H., 1936).

Le test des méthodes simples de gestion n'est pas très facile. En effet, les méthodes numériques susceptibles de fournir des solutions de référence optimales ne sont en mesure de traiter que des cas très simplifiés dans la modélisation hydrologique. Les méthodes comparées ici sont les suivantes :

- avec un modèle hydrologique raisonnable, on réalise une optimisation propre à chaque scénario généré, ou bien, on optimise à partir du seul scénario moyen; - avec un modèle hydrologique très simplifié, on peut établir une solution de référence par programmation dynamique.

Quel est donc le plus important pour les résultats opérationnels: représentation hydrologique ou qualité de l'outil mathématique utilisé ?

Les essais présentés ici n'ont pas l'ambition d'apporter une réponse générale à cette gestion, mais de contribuer aux réflexions en ce sens, tout en présentant des méthodes d'emploi très simples, susceptibles d'être utilisées sans difficulté par les gestionnaires.

\section{L'objectif de régularisation des débits}

L'objectif que nous nous proposons de satisfaire est de soutenir les étiages et d'écrêter les crues avec un réservoir dont la capacité utile est fixée a priori. Il s'agit donc d'une régularisation des débits à l'aval d'un point où existe un ouvrage. On entreprend de réaliser cette régularisation au pas de temps journalier. En période de crue, une gestion à pas de temps plus fin devrait être ajoutée à ce schéma journalier. (Nous n'aborderons pas ce problème dans le présent document).

(*) CERGRENE, ENPC, Noisy-le-Grand

(**) CEMAGREF, Antony.

\section{The operation of a reservoir by the means of discharge regulation}

In this paper we address the operation of a reservoir devoted both to low flow augmentation and to flood control. A single operation rule, using hydrological model based on simulation of scenarios for future flows, is developped and applied to a real case situation. Its results are compared to those given by a stationary stochastic dynamic programming method using a simplified hydrological process description. 


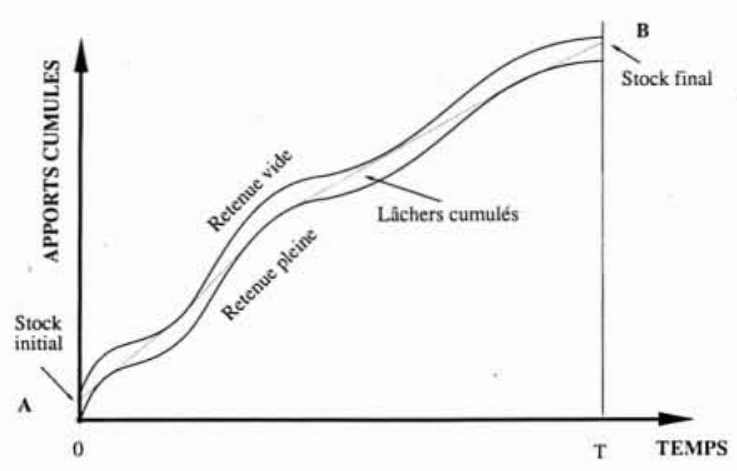

1. La méthode du fil-tendu.
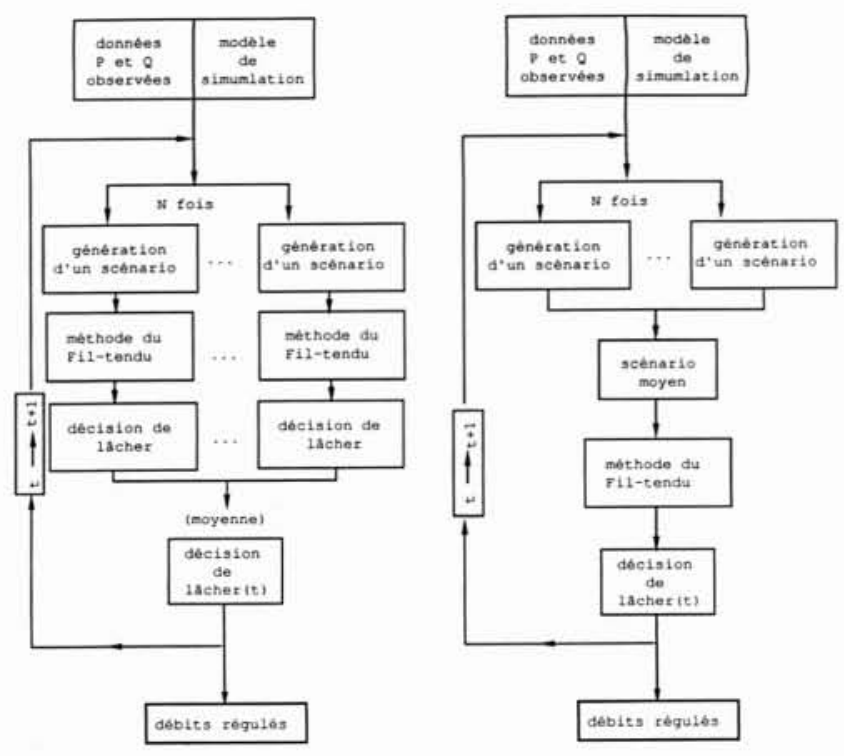

2. Schèmas alternatifs pour la méthode du fil-tendu sur scénarios simulés.
Soit $M$ le module du cours d'eau au point considéré ( $M$ est le débit moyen interannuel).

Soit $Q_{j}, j=1, n$, la série des débits naturels pour une période donnée. Désignons par $R_{j}, j=1, n$, la série des débits régularisés.

En fin de période, on peut calculer a posteriori (par la méthode du fil tendu exposée ci-après) quelle aurait été la meilleure gestion possible si l'on avait pu connaître a priori la série des $Q_{j}$ («optimum en avenir connu »). Soit $O_{j}, j=1, n$, la série des débits régulés de cette façon. C'est la limite physique des performances possibles de régulation offerte par le réservoir, c'est-à-dire la variance minimale des débits à l'aval du réservoir. En temps réel, la gestion ne peut donner que des résultats moins bons puisque l'on est confronté à la mauvaise connaissance des débits à venir. Un critère simple pour juger une méthode de régularisation est de mesurer le rapport de la réduction de la variance des débits obtenue par cette régulation, sur la réduction maximale idéale. On aura un critère $(C)$ compris entre $0 \%$ et $100 \%$ avec le rapport suivant :

$$
C=\frac{\sum_{i=1}^{n}\left(Q_{i}-M\right)^{2}-\sum_{i=1}^{n}\left(R_{i}-M\right)^{2}}{\sum_{i=1}^{n}\left(Q_{i}-M\right)^{2}-\sum_{i=1}^{n}\left(O_{j}-M\right)^{2}} .
$$

C'est ce critère que nous utiliserons dans la suite de ce document.

\section{La méthode du fil-tendu}

La méthode du «fil-tendu » est une méthode explicite de détermination des décisions de régulation optimale en avenir connu et doit son nom à la solution graphique introduite par H. VARLET (1966). Elle fournit une règle de gestion constante par morceaux, solution d'une gamme très large de critères à optimiser : il suffit que ce critère se présente sous la forme d'une somme de termes convexes stationnaires uniquement fonction des lâchers comme c'est le cas pour le critère quadratique ci-dessus.

On trace sur un graphique (temps, volume) la courbe des apports cumulés du bassin $\tilde{q}_{k}$ (fig. 1).

$$
\tilde{q}_{k}=\sum_{j=1}^{k} Q_{j} .
$$

On peut considérer $\tilde{q}_{k}$ comme le volume maximal que l'on peut recueillir à l'aval du réservoir entre les dates 1 et $k$, la retenue restant pleine. $\mathrm{Si}$ le réservoir a pour volume $V$, la courbe précédente translatée vers le haut d'une quantité $V$-donne la quantité maximale dont on peut disposer à l'aval du réservoir si on accepte de vider la retenue supposée initialement pleine. Ces deux courbes définissent un domaine de largeur constante $(V)$ pouvant représenter les états successifs du réservoir suite à une gestion donnée, le niveau de remplissage se mesurant depuis la courbe supérieure. Si l'on se fixe un niveau initial et un niveau final pour le réservoir, la méthode du "fil-tendu " permet de définir la politique optimale sous la forme de la série des stocks à maintenir dans le réservoir. Il suffit à cet effet de "tendre" un fil entre les 2 extrémités $A$ et $B$ de la figure 1 , en restant à l'intérieur du domaine défini précédemment.

Un algorithme assez rapide (KLEMES V., 1979) a été mis au point et intégré au logiciel que nous avons utilisé. 


\section{Méthode de gestion par application du fil-tendu sur de nombreux scénarios}

Puisque la gestion en avenir certain est résolue de façon très simple par l'algorithme du fil-tendu, on peut concevoir qu'une réponse acceptable au problème de la gestion en temps réel soit d'appliquer cet algorithme à plusieurs scénarios équiprobables permettant de balayer un large éventail d'avenirs possibles pour les écoulements du cours d'eau étudié, dans le même esprit que la programmation dynamique en avenir incertain simulé citée ci-dessus et avec les mêmes limitations. L'avantage est un allègement très sensible sur temps de calcul, rendant maniable sur un microordinateur cette technique plutôt réservée à des moyens puissants de calcul.

La méthode sera clairement explicitée lorsque l'on aura répondu aux trois questions suivantes:

a) combien de scénarios simuler à chaque pas de temps?

b) quelle durée donner à ces scénarios et quel stock objectif viser à la fin des scénarios ?

c) comment choisir une décision au vu de ces scénarios?

Nous allons décrire successivement ces points.

\subsection{Nombre de scénarios à simuler}

Le nombre de scénarios doit être assez important pour explorer convenablement la diversité des situations futures possibles. A priori, le nombre de scénarios à retenir varie avec le temps de réponse du bassin et l'importance de la réserve d'eau par rapport à l'écoulement annuel moyen. Si le temps de réponse du bassin est grand par rapport au pas de temps de la gestion (ici, le jour) alors le nombre de scénarios pourra être assez faible car, sur une durée où la situation n'aura guère évolué, on aura finalement simulé un nombre confortable de scénarios. Il en est de même si la réserve d'eau est très importante et donc se modifie lentement au fil des jours : le seul défaut sera alors une fluctuation des trajectoires, sans perte sensible de performance. Il faudra tester la méthode sur un grand nombre de bassins et de réservoirs pour dégager une règle quantitative pour la détermination d'un nombre souhaitable de scénarios. Pour l'instant on peut recommander de rechercher ce nombre de scénarios en l'augmentant progressivement par pas de 5 et en arrêtant quand le gain devient très faible et sans compensation pour la lourdeur des calculs exigés.

\subsection{Quelle durée donner aux scénarios et quelle condition finale adopter?}

La méthode de résolution du problème pour un scénario donné exige que l'on en fixe les conditions aux limites, c'est-à-dire la durée du scénario et le niveau dans le réservoir en fin de scénario. Etant donné que la variabilité des niveaux à l'intérieur d'une année est bien plus grande que la variabilité entre années, il semble judicieux que la durée d'un scénario soit un nombre entier d'années. On peut alors, sans trop se tromper, fixer un niveau final égal au niveau initial de remplissage. Si l'on désire tester l'intérêt de scénarios plus courts que l'année, il faut pouvoir proposer une règle de fixation du stock résiduel en fin de scénario. Cette tâche entraîne un supplément de calculs qui n'est pas compensé par la réduction des durées des scénarios (Yang X., 1989). On a donc retenu la solution plus simple d'une durée égale à un nombre entier d'années. Bien évidemment, plus la durée du scénario sera grande et moins la fixation du niveau final aura d'importance pour la décision présente, en début de scénario. Cependant le temps de calcul augmente proportionnellement et au-delà d'une certaine durée de scénario le gain marginal est quasi nul. Comme pour le nombre de scénarios, on devra déterminer la durée par essais successifs ( 1 an, 2 ans, rarement plus), en s'arrêtant dès que les résultats ne s'améliorent plus.

\subsection{Choix de la décision}

Chaque scénario, résolu par la méthode du "fil-tendu » conduit à la décision optimale qui lui est propre. On propose de choisir la décision en temps réel au vu de ces différentes décisions optimales particulières. Il est probable que l'on ne s'écartera pas beaucoup de l'optimum en adoptant la moyenne ou la médiane des décisions relatives aux différents scénarios. Une règle de choix plus complexe n'a pas été étudiée et on s'est limité à l'utilisation de la valeur moyenne.

\section{Méthode de gestion sur le scénario moyen}

Une alternative encore plus simple consiste à choisir comme décision celle qui est donnée par la méthode du fil-tendu appliquée au scénario moyen (moyenne, pour chaque pas de temps futur, des débits des différents scénarios simulés). Les deux démarches sont illustrées dans la figure 2.

\section{Elaboration des scénarios}

Le choix du modèle utilisé pour la génération des scénarios est indépendant de la méthode de gestion proposée. Cette séparation est d'ailleurs un avantage important de ce type de méthode. On présente la solution adoptée ici, ce qui nous permettra d'évoquer les difficultés rencontrées.

Il est en général assez délicat de modéliser directement les débits. Les modèles adoptés à cet effet ne sont pas très fidèles et restent d'un calage assez complexe, comme par exemple les modèles ARIMA saisonniers sur une transformée du débit. Un tel modèle statistique rustique sera utilisé, par la suite au $\S 10$. Par contre, une simulation des pluies suivie d'une transformation pluiedébit par un modèle conceptuel à réservoirs offre classiquement l'avantage de bâtir des scénarios cohérents avec les écoulements antérieurs observés. Le modèle de simulation de pluie est un modèle simple associant pour chaque mois calendaire une distribution de pluie journalière dépendante de 3 paramètres. On a adopté une loi de Weibull à seuil :

$$
F(x)=F_{0}+\left(1-F_{0}\right)\left[1-\mathrm{e}^{-\left(\frac{x}{a}\right)^{\alpha}}\right] \quad x \in(0,+\infty) .
$$




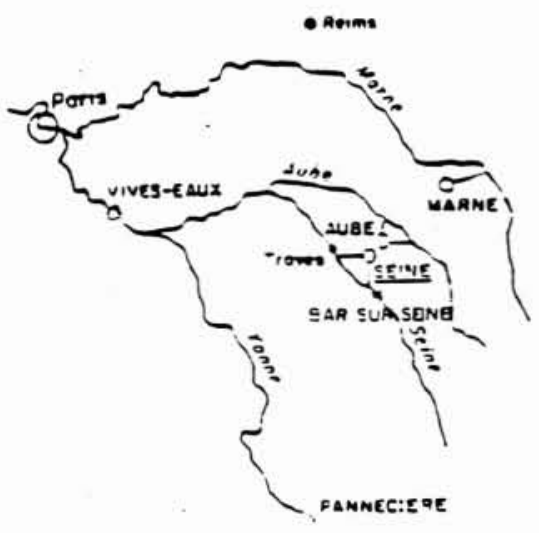

3. La Seine à Bar-sur-Seine.

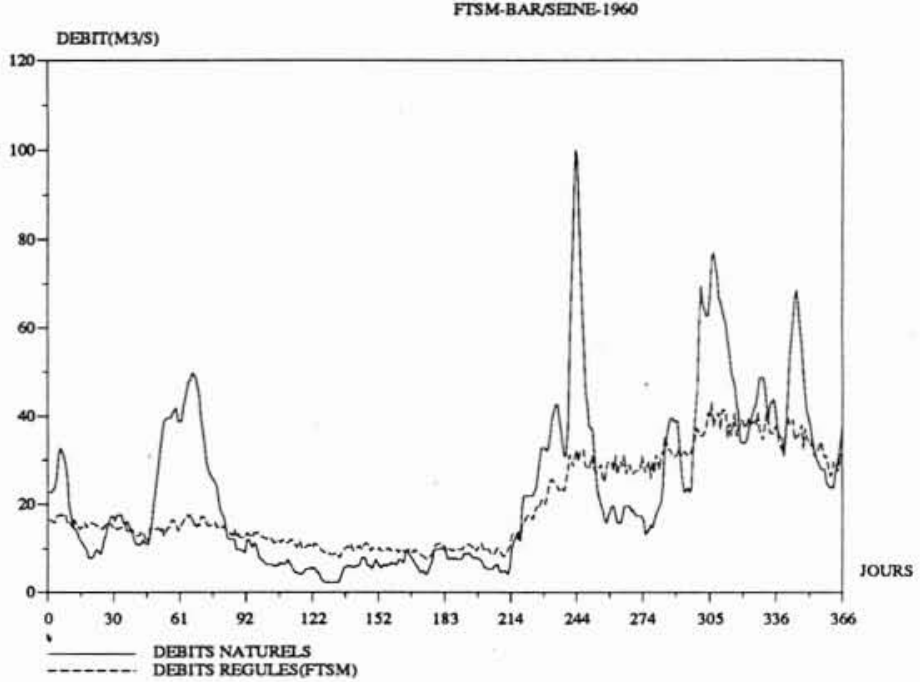

4. Aperşu sur l'année 1960 du contrôle de la méthode du filtendu sur scénarios moyens (FTSM).

Tableau 1

Influence du nombre et de la durée des scénarios sur les performances de la méthode

\begin{tabular}{|c|c|c|c|c|c|c|c|}
\hline$L$ & 1 & 2 & 3 & 5 & 7 & 10 & 15 \\
\hline 1 an & 82.4 & 87.7 & 89.1 & 89.2 & 90.5 & 90.5 & 90.8 \\
\hline 2 ans & 82.4 & 88.3 & 87.8 & 90.0 & 90.4 & 90.2 & 90.6 \\
\hline 3 ans & 83.2 & 87.6 & 88.2 & 90.0 & 90.1 & 90.4 & 90.8 \\
\hline 4 ans & 84.3 & 88.0 & 88.1 & 89.8 & 90.3 & 90.2 & 90.5 \\
\hline
\end{tabular}

Tableau 2

Nombre de scénarios et méthode du scénario moyen (durée d'un an)

\begin{tabular}{|c|c|c|c|c|c|c|c|c|}
\hline$N$ & 2 & 3 & 5 & 7 & 10 & 15 & 20 & 30 \\
\hline$C$ & 87.9 & 89.4 & 89.4 & 90.5 & 90.6 & 90.7 & 90.8 & 90.9 \\
\hline
\end{tabular}


Les trois paramètres $F_{0}, a, \alpha$ sont ajustés mensuellement par détermination du nombre moyen de jours sans pluie et par la méthode des moments.

Pour la simulation, on tire au hasard un nombre aléatoire $F$ compris entre 0 et 1 .

Si $F \leqslant F_{0}$, la pluie journalière est nulle.

Si $F>F_{0}$, la pluie vaut : $a\left(\ln \frac{1-F_{0}}{1-F}\right)^{1 / \alpha}$.

En ce qui concerne la transformation pluie-débit on a choisi le modèle GR3 (EdIJATNO, Michel C., 1989) qui est un exemple récent de modèle à réservoirs, dépendant de trois paramètres à caler.

Pour l'utiliser pour la génération de scénarios (c'est-àdire comme modèle de prévision), il convient de faire une mise à jour du modèle pour tenir compte de l'écoulement observé le jour du début du scénario. Pour simplifier, cette mise à jour porte uniquement sur l'état du réservoir eau gravitaire directement responsable des écoulements.

\section{Présentation du cas concret étudié (fig. 3)}

On s'intéresse à la régularisation des débits de la Seine à l'aval du réservoir de Bar-sur-Seine en amont de Troyes.

Le bassin versant de la Seine en amont de la dérivation vers le réservoir Seine a une superficie de $2340 \mathrm{~km}^{2}$. Le module est de $22 \mathrm{~m}^{3} / \mathrm{s}$. Les lois mensuelles de distribution des pluies journalières ont été ajustées sur 28 années de données. Les paramètres du modèle GR3 ont été calés sur les années 1972-1977.

\section{Choix du nombre et de la durée des scénarios}

On a testé diverses valeurs du nombre de scénarios et de la longueur de ceux-ci.

$$
N=\text { Nombre de scénarios : } 1,2,3,5,7,10,15
$$$$
L=\text { Longueur des scénarios : 1, 2, 3, } 4 \text { années. }
$$

Ces tests ont été réalisés sur la période 1975-1977 et ont donné les résultats qui apparaissent dans le tableau 1 . On y a représenté pour chaque couple $(N, L)$ la valeur du critère de régularisation adopté.

Des scénarios d'un an apparaissent suffisants dans ce cas. Une dizaine de scénarios semblent souhaitables, mais l'examen des graphiques montre une oscillation excessive des débits régulés. 30 scénarios ont été suffisants pour corriger cet effet.

Le temps calcul étant lié surtout à l'exécution de la méthode du fil-tendu, l'alternative qui consiste à utiliser cette méthode sur le scénario moyen est beaucoup plus rapide. Nous avons donc testé, à titre de comparaison, les gestions ainsi définies pour une durée des scénarios égale à une année et un nombre de scénarios allant jusqu'à 30 scénarios par jour. Les résultats apparaissent dans le tableau 2 et montrent que cette méthode, beaucoup plus rapide, donne des résultats équivalents à la précédente.

\section{Contrôle de la méthode sur une période différente}

Maintenant que le nombre et la durée des scénarios ont été fixés on peut contrôler la rusticité de la méthode proposée en l'appliquant sur une période différente de celle qui nous a permis d'en fixer les paramètres.

Les résultats ont été obtenus pour 3 ans de 1959 à 1961 : le critère est de $89,5 \%$, donc assez voisin de celui obtenu précédemment. La régularisation sur l'année 1960 apparaît sur la figure 4 . On peut dire que la méthode donne de bons résultats et se révèle d'une efficacité tout à fait honorable compte tenu de sa simplicité et de la rapidité de sa mise en œuvre.

\section{Comparaison avec une méthode de programmation dynamique stationnaire (PDS)}

Pour mieux situer les méthodes simples proposées, nous les avons comparées avec une méthode de programmation dynamique stochastique en régime stationnaire. Cependant une telle méthode est difficile à mettre en œuvre en utilisant le modèle GR3 dont l'état à un instant donné est caractérisé par les niveaux de 2 réservoirs. Pour une mise en œuvre commode de la programmation dynamique, il a été nécessaire de recourir à un simple modèle autorégressif sur les logarithmes des débits :

$$
\begin{gathered}
Y(t)=\log Q(t) \\
Y(t+1)=a(t)+b(t) Y(t)+\sigma(t) G(t)
\end{gathered}
$$

où les $N(t)$ sont des variables aléatoires indépendantes, normales centrées réduites (YANG X., 1989).

$\mathrm{Si}$ on admet que cette modélisation peut aussi être valable en temps continu, on obtient le processus de diffusion particulier :

$$
\mathrm{d} Y(t)=\{a(t)+(b(t)-1) Y(t)\} \mathrm{d} t+\sigma(t) \mathrm{d} B(t)
$$

où $B(t)$ est un processus de Wiener.

Le stock $S$ évolue, quant à lui, avec la loi :

$$
\begin{aligned}
\mathrm{d} S(t) / \mathrm{d} t=-u(t) \\
u_{\min } \leqslant u(t) \leqslant u_{\max } ; u(t) \geqslant-Q(t) \\
0 \leqslant S(t) \leqslant S_{\max } \\
R(t)=Q(t)+u(t) .
\end{aligned}
$$

On connaît $R(t)$ quand on décide du lâcher $u(t)$. Mis sous cette forme le problème est une recherche de contrôle optimal d'un processus de diffusion particulier, de vecteur d'état $(S(t), Y(t))$, dont la commande est $u(t)$. Le critère à optimiser est de la forme :

$$
V(S, Y, t)=\underset{u(t)}{\operatorname{Min}} E\left\{\int_{t}^{T}(R(\tau)-M)^{2} \mathrm{~d} \tau\right\} .
$$

Sous des conditions de régularité très générales l'équation ci-dessus est équivalente à l'équation différentielle de Hamilton-Jacobi : 


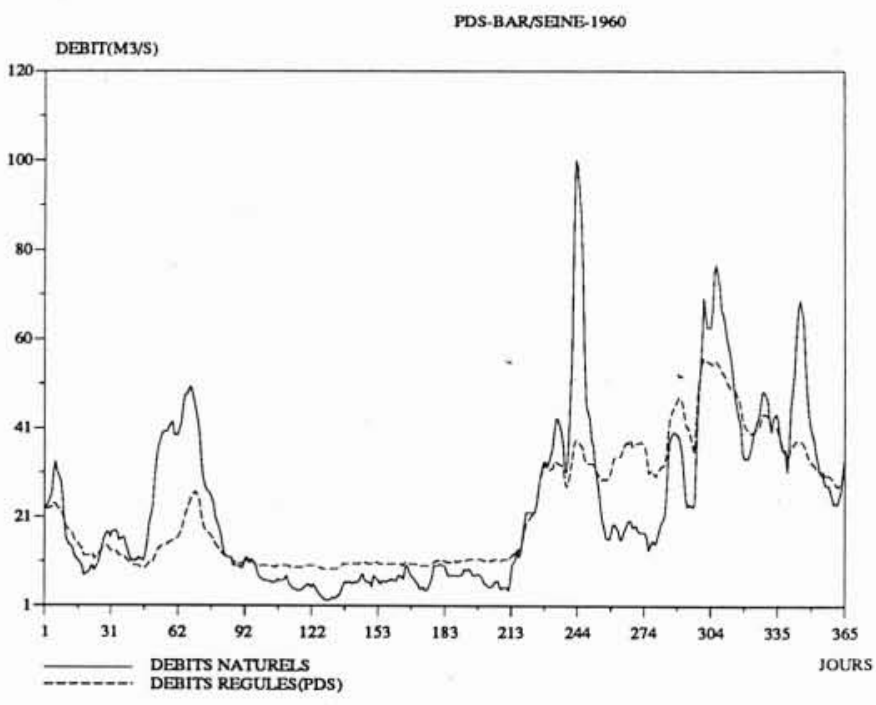

5. Résultats de la programmation dynamique stationnaire (PDS) sur l'année 1960.

$$
\begin{aligned}
\frac{\partial V}{\partial t}+\frac{\partial V}{\partial Y}\{a(t)+b(t) Y\}+\frac{1}{2} \sigma^{2}(t) \frac{\partial^{2} V}{\partial Y^{2}}= \\
=\underset{u}{\operatorname{Max}}\left\{\frac{\partial V}{\partial S} u-\left(M-e^{Y}-U\right)^{2}\right\} \\
u_{\min } \leqslant u \leqslant u_{\max } .
\end{aligned}
$$

La résolution fournit à la fois la solution du lâcher en feedback $u=u(S, Y, t)$ et la valeur de l'optimum du critère : $V(S, Y, t)$.

Pour transformer ce problème en temps continu en un problème discret journalier de commande d'une chaîne de Markov, on a utilisé une méthode de résolution explicite (KUSHNER, 1977), dans laquelle les transitions ne se font qu'entre états immédiatement voisins. Une telle technique a déjà été utilisée pour la gestion décadaire de la Seine en étiage (KATAI O., 1982).

\section{Application pratique et résultats numériques}

L'algorithme proposé par KUSHNER est stable numériquement sous les conditions suivantes: (DELEBECQUE F., Quadrat J. P., 1978) $0 \leqslant P_{0}(u) \leqslant 1$ soit

$$
\left\{\frac{|a(t)+(b(t)-1) Y(t)|}{\Delta Y}+\frac{|\sigma(t)|^{2}}{\Delta Y^{2}}+\frac{u_{\max }}{\Delta S}\right\} \leqslant \frac{1}{\Delta t} .
$$

Dans les calculs nous avons choisi $\Delta t=1$ jour

$$
\begin{aligned}
\frac{\Delta S}{S_{\max }} & =\frac{\Delta Y}{Y_{\max }}=\frac{1}{21} \text { avec } S_{\max }=200 \mathrm{Mm}^{3} \\
\text { et } Y_{\max } & =\log \left(Q_{\max }\right) \text { avec } Q_{\max }=100 \mathrm{~m}^{3} / \mathrm{s} .
\end{aligned}
$$

En fait ici l'objet de la gestion est de trouver des règles de gestion stationnaires de période 365 jours $u(S, Q, t)=u(S, Q, t+365)$ pour lesquelles le critère à optimiser aurait plutôt été

$$
\lim _{T \rightarrow \infty} \frac{1}{T-t} E\left[\int_{t}^{T}(R(\tau)-M)^{2} \mathrm{~d} \tau\right] .
$$

La formulation précédente permet une solution approchée à ce problème.

On fixe d'abord comme solution terminale $V(S, Q, 365)$ les valeurs obtenues par programmation dynamique déterministe sur les apports moyens puis on itère sur un nombre d'années suffisant pour que dans les calculs de $V(S, Q, t)$ et $u(S, Q, t)$ les solutions obtenues deviennent stationnaires.

Dans l'exemple ci-dessus trois années sont suffisantes pour obtenir une stationnarisation des résultats.

Pour calculer les performances on a appliqué la solution obtenue en feedback $u(S, Q, t), t=1$ janvier au 31 décembre sur la même période de validation 19591961.

Les résultats apparaissent sur la figure 5 et le tableau 3 et correspondent pour la période 59-61 à un critère de $85,4 \%$, c'est-à-dire sensiblement équivalent à celui obtenu avec la méthode du fil-tendu sur scénarios avec le modèle GR3, mais nettement supérieur à celui obtenu par cette méthode si l'on utilise comme générateur de

Tableau 3

Comparaison des différentes méthodes utilisées (test sur 1959-1961)

\begin{tabular}{|c|c|c|}
\hline Méthode utilisée & $\begin{array}{c}\text { Modèle } \\
\text { hydrologique }\end{array}$ & Efficacité \\
\hline $\begin{array}{c}\text { fil-tendu sur scénario moyen } \\
\text { (30 scénarios de 1 an) }\end{array}$ & GR3 & $69.5 \%$ \\
\hline $\begin{array}{l}\text { fil-tendu sur scénario moyen } \\
(30 \text { scénarios de 1 an) }\end{array}$ & autorégressif & $62.2 \%$ \\
\hline programmation dynamique stationnaire & autorégressif & $87.4 \%$ \\
\hline
\end{tabular}



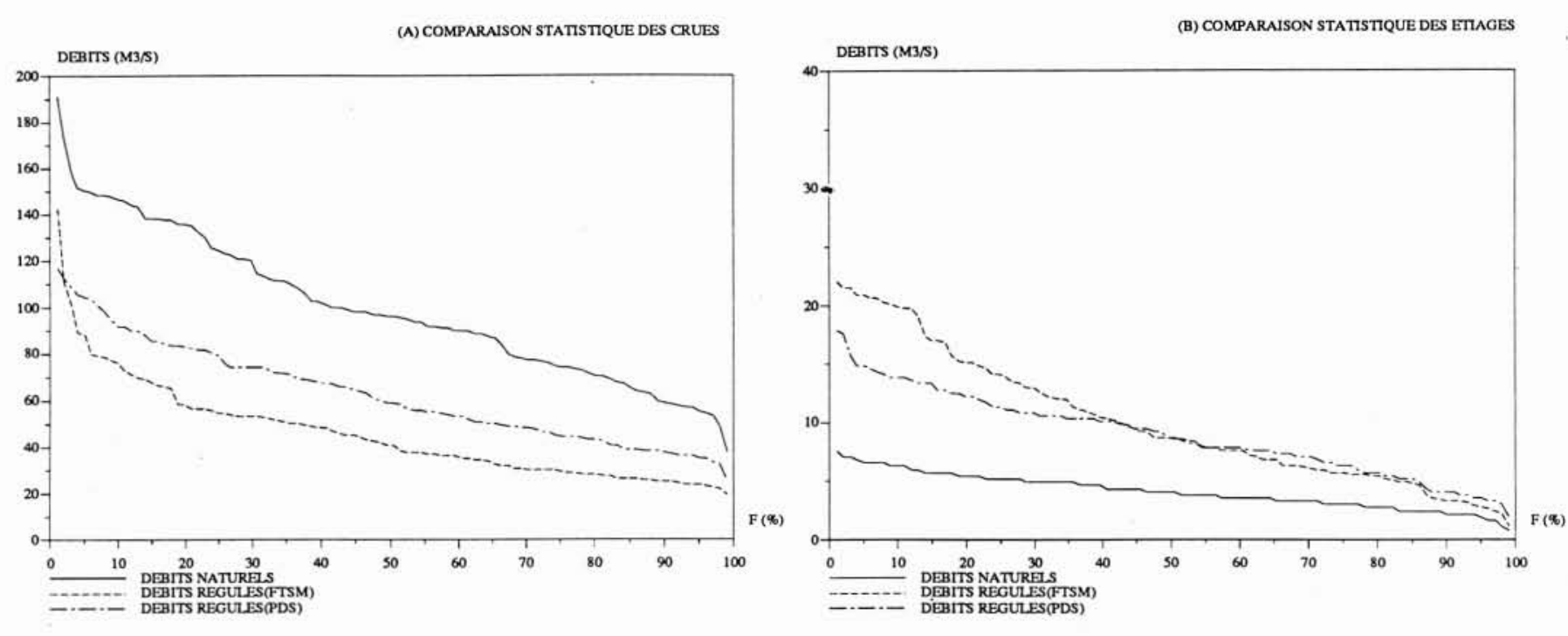

6. Comparaison du fil-tendu sur scénarios moyens (FTSM) et de la programmation dynamique stationnaire (PDS) sur les distributions des crues $(A)$ et des étiages (B).

scénarios le modèle autorégressif employé pour la programmation dynamique.

Une comparaison également intéressante concerne l'effet de la réserve sur les distributions des étiages et des crues.

A cet effet, une période de 100 années de débits journaliers a été simulée à l'aide du modèle GR3 et les distributions du maximum et du minimum annuels ont été établies. Puis la gestion du réservoir de Bar-sur-Seine a été effectuée sur cette même période par la méthode du fil-tendu sur scénario moyen et par la programmation dynamique avec discrétisation selon la méthode de KUSHNER. Les distributions du maximum et du minimum annuels pour deux séries de débits régulés ont été reportées sur les graphiques de la figure 6. Les deux méthodes donnent des résultats semblables en ce qui concerne les étiages avec un léger avantage à la programmation dynamique. La méthode du fil-tendu sur scénario moyen semble sensiblement plus efficace pour l'écrêtement des crues. Globalement, sur cette longue série synthétique générée par le modèle GR3, le critère d'efficacité présenté dans le $\$ 2$ est en faveur de la méthode du fil-tendu, $88,8 \%$, contre $75,0 \%$ pour la programmation dynamique en avenir incertain (avec un modèle hydrologique rudimentaire).

\section{Conclusion}

On a proposé une méthode simple pour la gestion d'un réservoir destiné à la régulation des débits. Cette méthode correspond à l'utilisation de la technique du filtendu sur un scénario moyen obtenu à partir d'une trentaine de scénarios générés par le modèle GR3. Le recours à un modèle hydrologique permet à cette méthode de supporter la comparaison avec une méthode de programmation dynamique en régime stationnaire, théoriquement plus optimale, mais s'appuyant en pratique nécessairement sur un modèle hydrologique plus pauvre. L'introduction d'une optimisation pour chaque scénario n'a pas apporté d'amélioration des performances, vraisemblablement parce que le choix de la décision moyenne adopté ici est trop simpliste. Un travail en ce sens est en cours au CERGRENE. Enfin cette méthode de gestion simplifiée est dépendante de la qualité du modèle hydrologique avec lequel elle est couplée: avec le même modèle que celui associé à la programmation dynamique, ses performances chutent. Elle s'adresse donc prioritairement aux hydrologues qui $\mathrm{y}$ trouveront un outil standard pouvant valoriser de façon immédiate tout progrès fait dans la prévision des débits à court et moyen terme, sans nécessiter de programmation particulière en cas considéré. 


\section{Références}

Delebecque F., Quadrat J. P. : " An Example of Large Scale System: Management of Hydropower Production, Transactions on Automatic Control ", IEEE, April 1978.

EdiJATNo, Michel C. : «Un modèle pluie-débit journalier à trois paramètres ", La Houille Blanche, n" 2, 1989.

Goussebaile J., Miquel J., Roche P. A. : "Gestion des barrages réservoirs en avenir incertain : utilisation des méthodes d'optimisation en avenir certain sur scénarios", SHF, XIX $X^{e s}$ Journées de l'hydraulique, Paris 1986, gestion No. V, rapport n' 11.

KATAI O.: " Optimal Control of Discharge from a Reservoir Based on Finite Kushner's Markov Chain Approximation of River Flow Diffusion Process ", Research Report, Faculty of Engineering, Kyoto University, Kyoto, Japan, 1982.

Klemes V. : « Storage Mass-Curve Analysis in a System-Analytic Perspective ", Water Resources Research, Vol. 15, April 1979.

Kushner Harold J.: "Probability Methods for Approximations in Stochastic Control and for Elliptic Equations ", Mathematics in Science and Engineering, Volume 129, Academic Press, 1977.

Miquel J., Roche P. A. : " La gestion d'un réservoir de soutien d'étiages peut-elle être optimale en cas de prévisions imparfaites?", Scientific procedures applied to the planning, design and management of water resource systems, (Proceedings of the Hamburg Symposium, août 1983) IAHS, Publ. No. 147.

VARLET H. : "Barrage-Réservoir ", Tome 1, Eyrolles, 1966.

YANG X., "Gestion des barrages-réservoirs en avenir incertain : utilisation de la méthode du fil-tendu sur scénarios". rapport de DEA-TGE, Paris XII, ENPC-ENGREF, 1989. 